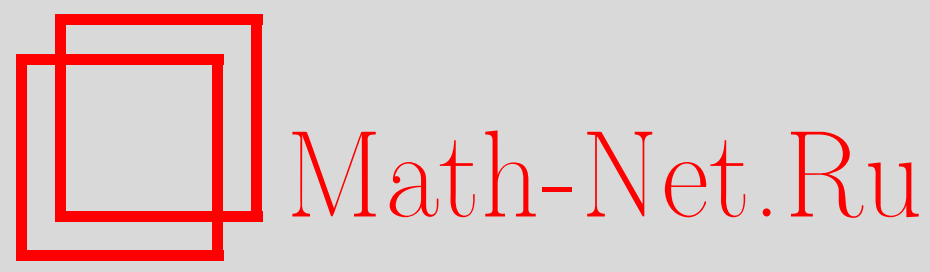

Л. А. Калякин, Ю. Ю. Багдерина, Асимптотика ограниченных на бесконечности решений уравнений квадратичного главного резонанса, Матем. заметки, 2005, том 78, выпуск 1, 85-97

DOI: https://doi.org/10.4213/mzm2564

Использование Общероссийского математического портала Math-Net.Ru подразумевает, что вы прочитали и согласны с пользовательским соглашением http://www . mathnet.ru/rus/agreement

Параметры загрузки:

IP : 54.80 .73 .141

26 апреля 2023 г., $16: 31: 54$

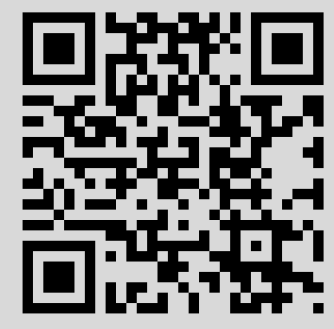




\title{
АСИМПТОТИКА ОГРАНИЧЕННЫХ \\ НА БЕСКОНЕЧНОСТИ РЕШЕНИЙ УРАВНЕНИЙ КВАДРАТИЧНОГО ГЛАВНОГО РЕЗОНАНСА
}

\author{
Л. А. Калякин, Ю. Ю. Багдерина
}

\begin{abstract}
Рассматривается система шести нелинейных дифференциальных уравнений, которая возникает при усреднении быстрых вынужденных колебаний. Основной результат состоит в построении асимптотики на бесконечности для общего решения с ограниченньми амплитудами. Выяснено, что структура асимптотических рядов зависит от параметров так, что коэффициенты рядов меняются скачком на резонансном множестве.

Библиографоия: 17 названий.
\end{abstract}

Рассматривается нелинейная неавтономная система с тремя степенями свободы, записанная в переменных амплитуда-фаза:

$$
\begin{aligned}
& \frac{d \rho_{j}}{d \theta}+\gamma_{j} \rho_{k} \rho_{l} \sin \psi=f_{j} \sin \left(\psi_{j}+\varphi_{j}\right), \quad \rho_{j} \frac{d \psi_{j}}{d \theta}+\gamma_{j} \rho_{k} \rho_{l} \cos \psi=f_{j} \cos \left(\psi_{j}+\varphi_{j}\right) \\
& \psi=\psi_{1}+\psi_{2}+\psi_{3}, \quad \gamma_{1}=1, \quad \gamma_{2}=\gamma_{3}=-1, \quad j=1,2,3, \quad j+k+l=6, \quad k \neq l
\end{aligned}
$$

Характерной чертой этих уравнений является квадратичньй рост на бесконечности заданных фазовых функций: $\varphi_{j}(\theta)=\omega_{j} \theta+\alpha_{j} \theta^{2}, \alpha_{j}, \omega_{j}, f_{j}=$ const; $\alpha_{j} \neq 0$ при $f_{j} \neq 0$. Основная цель работы - построение асимптотики на бесконечности для общего (шестипараметрического) решения, в котором амплитуды $\rho_{j}(\theta)$ остаются ограниченными при $\theta \rightarrow \infty$.

Если каждую пару переменньх $\rho_{j}, \psi_{j}$ рассматривать как полярные координаты на плоскости, то в соответствующих декартовых координатах $x_{j}=\rho_{j} \cos \psi_{j}, y_{j}=\rho_{j} \sin \psi_{j}$ уравнения принимают вид

$$
\frac{d x_{j}}{d \theta}+\gamma_{j}\left(x_{k} y_{l}+y_{k} x_{l}\right)=f_{j} \sin \varphi_{j}, \quad \frac{d y_{j}}{d \theta}+\gamma_{j}\left(x_{k} x_{l}-y_{k} y_{l}\right)=f_{j} \cos \varphi_{j}
$$

Однако при построении асимптотик удобнее пользоваться уравнениями (1), рассматривая их в замкнутой области шестимерного пространства $\mathbb{R}_{+}^{6}=\left\{\rho_{j} \geqslant 0, \psi_{j} \in \mathbb{R}\right\}$. При аккуратной постановке задачи дифференциальные уравнения в форме (1) должны

Работа выполнена при поддержке Российского фонда фундаментальных исследований, гранты № 03-01-00716 и № 04-02-97503, программы “Ведущие научные шшколы", грант № 1446.2003.1, и фонда INTAS, грант № 03-51-4286. 
быть дополнены условиями скачка для фаз при переходе амплитудой нулевого значения: $\psi_{j}(\theta+0)-\psi_{j}(\theta-0)=\pi$ при $\rho_{j}(\theta)=0$. В ситуации общего положения амплитуды отличны от нуля в главньх членах асимптотики и условия скачка не используются.

Происхождение задачи. Интерес к системам (1) объясняется их ролью в теории нелинейных колебаний. Хотя уравнения представляются весьма специфическими, они являются стандартньми при описании резонансных явлений для колебательных процессов разной природы [1]-[3]. Обычно уравнения такого типа возникают при усреднении по быстрьм колебаниям других, более сложных систем [4]. Искомые функции $\rho_{j}(\theta), \psi_{j}(\theta)$ представляют собой медленно меняющиеся амплитуды и сдвиги фаз быстрых гармонических колебаний. Нелинейности исходных систем индуцируют нелинейности в усредненных уравнениях посредством главных резонансов. В частности, квадратичные резонансы приводят к квадратичным нелинейностям, указанным в (1). Неавтономность уравнений (1) является следствием медленной деформации параметров исходных систем, таких, как частоты собственньх колебаний, частоты и амплитуды внешних сил. Как раз эффект деформации вынуждающих частот для ситуации общего положения содержится в квадратичных слагаемых фазовых функций $\varphi_{j} \approx \theta^{2}$. Примеры такого типа многомерных систем приведены в [5], [6].

Постановка вопроса об асимптотике по медленному времени $\theta \rightarrow \infty$ связана с использованием усредненных уравнений (1) для описания переходных процессов, например, нелинейного резонанса или начального этапа авторезонанса (автофазировки) [7]-[9]. Рассматриваемая здесь система, по-видимому, является простейшей среди многомерных систем, описывающих суть переходных резонансных явлений. Мы намеренно ограничиваемся уравнениями в максимально простой форме, чтобы выявить основные отличия от одномерного случая [10]. Всевозможные обобщения с более сложной структурой правых частей не вносят принципиальных отличий.

С многомерньми нелинейными колебаниями часто ассоциируется проблема малых знаменателей и возникновение хаоса. Рассматриваемые нами решения не содержат таких эффектов. Отсутствие малых знаменателей в предъявляемых конструкциях является следствием конечного числа гармоник в накачке (в правых частях уравнений).

Постановка задачи об асимптотике решений на бесконечности является классической, и на эту тему имеется огромное число публикаций. Современное состояние общей теории освещено в монографиях [11], [12]. Однако известные результаты непосредственно к уравнениям (1) не применимы из-за осциллирующего характера решений. Используемые нами приемы состоят в модификации разных вариантов нелинейного метода ВКБ [13], [14]. Основной целью является исследование общего шестипараметрического решения уравнений (1), для которого строится асимптотическое разложение при $\theta \rightarrow \infty$. Следует подчеркнуть, что получаемые асимптотики не являются равномерными по параметрам. Более того, в пространстве параметров имеется исключительное множество, которое связано с резонансами. При выходе параметров на резонансноемножество меняется структура асимптотики. Хотя мера такого (резонансного) множества равна нулю, но оно всюду плотно. Ситуация здесь схожа с КАМ теорией и с усреднением многомерных возмущенных систем [15], [16]. Однако надо иметь ввиду, что обсуждаемая задача об асимптотике решения на бесконечности по своей постановке не имеет прямого отношения ни к КАМ теории, ни к задачам с малым параметром. Рассматриваемые решения не являются условно периодическими и не содержат малых параметров.

Вопрос об асимптотиках, равномерных по константам интегрирования, остается от- 
крытым. В данной работе эта проблема обходится следуюшим образом. Обрьв бесконечного ряда приводит к расширению области равномерности асимптотики. Расплатой за расширение множества параметров становится конечньй порядок аппроксимации. Чем вьше порядок аппроксимации, тем шире множество исключительных параметров. Для асимптотик с конечным порядком аппроксимации множество подходящих параметров оказывается открытым. В задаче с одной степенью свободы таких эффектов не наблюдается [10].

Помимо рассматриваемых здесь решений, которые остаются ограниченньми при $\theta \rightarrow \infty$, существуют решения, неограниченно растущие при $\theta \rightarrow \infty$. Их анализ выходит за рамки данной заметки и будет приведен в другой публикации. Заметим лишь, что параметры из резонансного множества не имеют отношения к растушим решениям. На резонансном множестве меняются скачком лишь члены старших порядков в предъявляемых ниже асимптотических рядах.

1. Невозмущенная система. Основой для предлагаемых ниже асимптотических конструкций служат решения автономной системы, которая получается из (1) при $f_{j} \equiv 0$. Такая система

$$
\frac{d \rho_{j}}{d \theta}+\gamma_{j} \rho_{k} \rho_{l} \sin \psi=0, \quad \rho_{j} \frac{d \psi_{j}}{d \theta}+\gamma_{j} \rho_{k} \rho_{l} \cos \psi=0
$$

при $\psi=\psi_{1}+\psi_{2}+\psi_{3}, \gamma_{1}=1, \gamma_{2}=\gamma_{3}=-1, j=1,2,3, j+k+l=6, k \neq l$, называемая далее невозмущенной, легко интегрируется.

ТЕорема 1. Для системы уравнений (3) существует общее шестипараметрическое решение

$$
\rho_{j}=\stackrel{0}{R}_{j}\left(\theta+S_{0} ; \mathbf{E}\right), \quad \psi_{j}=\stackrel{0}{\Psi}_{j}\left(\theta+S_{0} ; \mathbf{E}\right)+\theta \Omega_{j}(\mathbf{E})+S_{j}, \quad j=1,2,3,
$$

с шестью произвольным ми константами: $\mathbf{E}=\left\{E_{1}, E_{2}, E_{3}\right\}, \mathbf{S}=\left\{S_{0}, S_{1}, S_{2}\right\}$ при $S_{1}+S_{2}+S_{3}=0$. Вектор-функиии $\stackrel{0}{R}_{j}(\theta ; \mathbf{E}), \stackrel{0}{\Psi}_{j}(\theta ; \mathbf{E})$ являются гладкими, периодическими по $\theta$ с периодом $\Theta=\Theta(\mathbf{E})$ и с частотой $\Omega=2 \pi / \Theta$. В фазовых функииях $\psi_{j}$ содержатся линейные по $\theta$ слагаемые с коэффициентами, которые удовлетворяют соотношению $\Omega_{1}+\Omega_{2}+\Omega_{3} \equiv 0$.

ДополнЕниЕ. Все остальные решения невозмущенной системы либо входят в четырехпараметрическое семейство решений с постоянными ненулевыми амплитудами $R_{1}=$ $R_{2} R_{3} / \sqrt{R_{2}^{2}+R_{3}^{2}} \forall R_{2}, R_{3}=$ const $\neq 0$, либо входят в четырехпараметрическое семейство непериодических решений, либо представляют собой неподвижные точки (для системы в декартовых координатах типа (2)), в которых, по крайней мере, пара амплитуд обращается в нуль.

ЗАМЕчАнИЕ. В случае, когда в исходных уравнениях все коэффициенты $\gamma_{j}=1$ одного знака, любые нетривиальные решения имеют полюса. Такие уравнения здесь не рассматриваются.

ДокАЗАТЕЛЬСТво теоремы основано на наличии трех первых интегралов:

$$
\rho_{1} \rho_{2} \rho_{3} \cos \psi=E_{1}, \quad \rho_{2}^{2}+\rho_{1}^{2}=E_{2}, \quad \rho_{3}^{2}+\rho_{1}^{2}=E_{3}
$$


значения которых используются в качестве параметров. Поскольку уравнения для четырех функций $\psi, \rho_{j}, j=1,2,3$, можно отделить от остальных:

$$
\rho_{j}^{\prime}+\gamma_{j} \rho_{k} \rho_{l} \sin \psi=0, \quad \rho_{1} \rho_{2} \rho_{3} \psi^{\prime}+\left[\left(\rho_{2} \rho_{3}\right)^{2}-\left(\rho_{1} \rho_{3}\right)^{2}-\left(\rho_{1} \rho_{2}\right)^{2}\right] \cos \psi=0,
$$

первые интегралы обеспечивают решение задачи в терминах эллиптических функций Якоби. Например, выражение одной из компонент через функцию синус амплитуды имеет вид

$$
\stackrel{0}{R}_{1}(\theta ; \mathbf{E})=\left[\eta_{1}+\left(\eta_{3}-\eta_{1}\right) k^{2} \mathrm{sn}^{2}\left(\sqrt{\eta_{3}-\eta_{1}} \theta, k\right)\right]^{1 / 2}, \quad k^{2}=\frac{\eta_{2}-\eta_{1}}{\eta_{3}-\eta_{1}}
$$

Используемые здесь параметры $\eta_{1}<\eta_{2}<\eta_{3}$ являются корнями полинома $\eta\left(E_{2}-\eta\right) \times$ $\left(E_{3}-\eta\right)-E_{1}^{2}$. В терминах этой эллиптической функции выписываются остальные компоненты решения $\rho_{j}, \psi_{j}$.

При интегрировании уравнений для фазовых функций появляются непериодические слагаемые, обусловленные ненулевьпи средними значениями

$$
\left.\left.\Omega_{j}=-\gamma_{j} E_{1} \frac{1}{\Theta} \int_{0}^{\Theta}(\stackrel{0}{R})_{j}\right)^{-2} d \theta \equiv-\gamma_{j} E_{1}\left\langle(\stackrel{0}{R})_{j}\right)^{-2}\right\rangle
$$

Очевидно, коэффициенты $\Omega_{j} \neq 0$ при $E_{1} \neq 0$. Однако сумма их равна нулю $\Omega_{1}+\Omega_{2}+$ $\Omega_{3}=0$, поскольку комбинационная фаза $\psi=\psi_{1}+\psi_{2}+\psi_{3}$ является периодической функцией. По той же причине три константы интегрирования $S_{j}$ содержат лишш два произвола; связь между ними определяется способом фиксации средних значений фаз $\psi_{j}$. Например, если осциллирующие части фиксировать, требуя нулевого среднего значения $\left\langle\stackrel{0}{\Psi}_{j}\right\rangle=0$, то константы интегрирования будут связаны со средним значением комбинационной фазы соотношением $S_{1}+S_{2}+S_{3}=\langle\psi\rangle$. Если же положить $S_{1}+S_{2}+S_{3}=0$, то среднее $\langle\psi\rangle$ будет содержаться в средних значениях осциллирующих частей $\stackrel{0}{\Psi}_{j}$. Теорема доказана.

В декартовых переменных полученное решение представляется трех-периодическими функциями $x_{j}(\theta), y_{j}(\theta)$ с частотами $\Omega, \Omega_{j}$. Заметим, что все амплитуды $\rho_{j}(\theta)$ имеют одну (основную) частоту $\Omega(\mathbf{E})$. Дополнительные частоты $\Omega_{j}(\mathbf{E})$ проявляются в компонентах $x_{j}(\theta), y_{j}(\theta)$ через фазовые функции.

На плоскости параметров $E_{2}=E_{3}$ при $E_{1} \neq 0$ происходит вырождение решения в двухчастотное с $\Omega_{2}=\Omega_{3}$ и $\rho_{2}(\theta) \equiv \rho_{3}(\theta)$. На плоскости параметров $E_{1}=0$ при $E_{2} \neq E_{3}$ решение вырождается в одночастотное с $\Omega \neq 0, \Omega_{j}=0$; в этом решении амплитуда $\rho_{1}(\theta)$ проходит через нулевое значение при постоянной комбинационной фазе $\psi \equiv \pm \pi / 2$. На пересечении плоскостей решения будут непериодическими. В данной работе анализируются решения со значениями параметров $\mathbf{E}$ вне этих двух плоскостей.

В структуре асимптотики важную роль играют резонансные соотношения для часTOT

$$
m \Omega(\mathbf{E})+p\left[\omega_{1}+\Omega_{1}(\mathbf{E})\right]+q\left[\omega_{2}+\Omega_{2}(\mathbf{E})\right]+r\left[\omega_{3}+\Omega_{3}(\mathbf{E})\right]=0, \quad m, p, q, r \in \mathbb{Z} .
$$

Эти соотношения определяют в пространстве параметров $\mathbf{E} \in \mathbb{R}^{3}$ поверхности, называемые резонансными [15]. Множество всех резонансных значений параметров $\mathbf{E}$ всюду плотно в области, где определены частоты $\Omega(\mathbf{E}), \Omega_{j}(\mathbf{E})$. Если ограничить номера 
$|p|,|q|,|r| \leqslant N<\infty$, то при $m \in \mathbb{Z}$ счетное множество резонансных поверхностей будет “довольно редким", так что дополнение к ним оказывается открытым множеством. Объединение таких резонансных поверхностей будет обозначаться через $\mathscr{E}_{N}$.

В пространстве параметров $\mathbf{E}$ имеется еще одно исключительное множество, при выходе на которое меняется структура асимптотики возмущенного решения. Оно состоит из точек, в которых частоты оказываются функционально зависимьми. Из аналитических свойств невозмущенного решения вытекает, что таких точек "не много":

СледСтвиЕ 1. Для матричы, составленной из градиентов частот $\partial\left(\Omega, \Omega_{1}, \Omega_{2}\right.$, $\left.\Omega_{3}\right) / \partial(\mathbf{E})$, ранг зависит от параметров и вне плоскостей $E_{1}=0, E_{2}=E_{3}$ его значение $r=r(\mathbf{E}) \geqslant 2 ;$ максимальный ранг $r=3$ всюду вне поверхности

$$
\mathscr{E}_{0}=\left\{\mathbf{E} \in \mathbb{R}^{3}: \operatorname{det} \frac{\partial\left(\Omega, \Omega_{k}, \Omega_{l}\right)}{\partial(\mathbf{E})}=0 \forall k \neq l\right\} .
$$

2. Линеаризованная система уравнений. Построение асимптотик на основе решения невозмущенной системы, как обычно, приводит к линеаризованньм уравненияM

$$
\begin{gathered}
\frac{d R_{j}}{d \theta}+\gamma_{j}\left[\stackrel{0}{R_{k}} \stackrel{0}{R_{l}} \cos \stackrel{0}{\Psi}\right] \Psi+\gamma_{j}\left[R_{k} \stackrel{0}{R_{l}}+\stackrel{0}{R}_{k} R_{l}\right] \sin \stackrel{0}{\Psi}=H_{\rho, j}(\theta), \\
\frac{d \Psi_{j}}{d \theta}-\gamma_{j} \Psi\left[\stackrel{0}{R}_{k} \stackrel{0}{R}_{l} \sin \stackrel{0}{\Psi}\right] / \stackrel{0}{R}_{j}+\gamma_{j} \cos \stackrel{0}{\Psi}\left[R_{k} \stackrel{0}{R}_{l}+R_{l} \stackrel{0}{R}_{k}-R_{j} \stackrel{0}{R}_{k} \stackrel{0}{R}_{l} / \stackrel{0}{R}_{j}\right] / \stackrel{0}{R}_{j}=H_{\psi, j}(\theta) \\
\Psi=\Psi_{1}+\Psi_{2}+\Psi_{3}, \quad j=1,2,3, \quad j+k+l=6 .
\end{gathered}
$$

В рассматриваемых ниже ситуациях правые части будут условно периодическими по $\theta$ функциями. Однако решение неоднородных уравнений $\left\{R_{j}, \Psi_{j}\right\}$ может оказаться неограниченньм. Для ограниченности решения требуются дополнительные условия на вектор-функции правых частей $\mathbf{H}=\left\{H_{\rho, 1}, H_{\rho, 2}, H_{\rho, 3}, H_{\psi, 1}, H_{\psi, 2}, H_{\psi, 3}\right\}^{\top}$. Такие условия выявляются в данном разделе.

Теорема 2. Пусть вектор-столбеи,

$$
\stackrel{0}{\mathbf{Y}}(\theta ; \mathbf{E}, \mathbf{S})=\left\{\stackrel{0}{R}_{j}\left(\theta+S_{0} ; \mathbf{E}\right), \stackrel{0}{\Psi}_{j}\left(\theta+S_{0} ; \mathbf{E}\right)+\theta \Omega_{j}(\mathbf{E})+S_{j}\right\}^{\top}
$$

представляет собой построенное выше шестипараметрическое решение нелинейной системь (3) вне плоскостей $E_{1}=0 u E_{2}=E_{3}$. Тогда для линеаризованной однородной системы, соответствующей (8), существует фундаментальная матрича решений $\mathscr{W}$, столбиы которой имеют следующую структуру:

$$
\mathbf{Y}_{i}(\theta), \quad i=0,1,2, \quad \mathbf{Y}_{i}(\theta)=\widehat{\mathbf{Y}}_{i}(\theta)+\theta \sum_{m=0}^{2} \nu_{i, m} \mathbf{Y}_{m}(\theta), \quad i=3,4,5
$$

Вектор-функиии $\mathbf{Y}_{i}(\theta), i=0,1,2, u \widehat{\mathbf{Y}}_{i}(\theta), i=3,4,5$, являются периодическими по $\theta$ с частотой $\Omega(\mathbf{E})$. Число линейно независимых непериодических решений равно $r$ - рангу матрицы $\partial\left(\Omega, \Omega_{1}, \Omega_{2}, \Omega_{3}\right) / \partial(\mathbf{E})$, через әлементы которой выражсаются коэффициенты $\nu_{i, m}=$ const. 
ДокАЗАТЕЛЬСтво. Одна тройка линейно независимых решений получается при дифференцировании вектор-функции $\stackrel{0}{\mathbf{Y}}(\theta ; \mathbf{E}, \mathbf{S})$ по переменным $S_{i}$ и имеет вид

$$
\mathbf{Y}_{0}=\partial_{\theta}\left\{\stackrel{0}{R}_{j}, \stackrel{0}{\Psi}_{j}\right\}^{\top}, \quad \mathbf{Y}_{1}=(0,0,0,1,0,-1)^{\top}, \quad \mathbf{Y}_{2}=(0,0,0,0,1,-1)^{\top}
$$

Другая тройка линейно независимых решений получается при дифференцировании по параметрам E:

$$
\mathbf{Y}_{i}=\partial_{E_{i-2}} \stackrel{0}{\mathbf{Y}}=\widehat{\mathbf{Y}}_{i}(\theta)+\theta \sum_{m=0}^{2} \nu_{i, m} \mathbf{Y}_{m}(\theta), \quad \nu_{i, m}=\text { const }, \quad i=3,4,5
$$

Периодическая часть здесь выделена в $\widehat{\mathbf{Y}}_{i}(\theta)$. Непериодические слагаемые обязаны зависимости частот от параметров $\mathbf{E}$; ранг матрищы $\left\{\nu_{i, m}\right\}$ равен $r$. Легко понять, что построенные таким образом решения будут линейно независимыми. Вронскиан вычисляется через след матрицы линеаризованной системы и для определенной выше системы решений выражается по формуле $\operatorname{det} \mathscr{W}=-\left(4 \stackrel{0}{R_{1}} \stackrel{0}{R}_{2} \stackrel{0}{R}_{3}\right)^{-1}$.

Наличие фундаментальной системы решений позволяет вьписать решения соответствующих неоднородных уравнений через интегралы

$$
\mathbf{Y}(\theta)=\mathscr{W}(\theta) \int_{\theta_{0}}^{\theta} \mathscr{W}^{-1}(\vartheta) \mathbf{H}(\vartheta) d \vartheta, \quad \theta_{0}=\text { const }
$$

Получаемое по этой формуле решение, вообще говоря, не будет ограниченным при $\theta \rightarrow \infty$, даже для периодической правой части Н. Ниже будут сформулированы условия ограниченности и предъявлена конструкция общего условно периодического решения. Поскольку в этой процедуре используется метод вариации произвольных постоянных, то основньпи объектами являются определители $W_{i}[\mathbf{H}]$, которые получаются из вронскиана $W=\operatorname{det} \mathscr{W}$ заменой $i$-го столбца $\mathbf{Y}_{i}$ на столбец правых частей $\mathbf{H}$. Чтобы не затемнять существо дела, ограничимся ситуацией общего положения, рассматривая те значения параметров $\mathbf{E}$, где частоты функционально независимы, т.е. при $r=3$.

ТЕорема 3. Пусть значения параметров $\mathbf{E}$ не принадлежат плоскостям $E_{1}=0, E_{2}=E_{3}$ и число непериодических базисных решений $r=3$. Если вектор-функиия правых частей $\mathbf{H}(\theta)$ имеет период $\Theta$, совпадающий с периодом решения нелинейной системы, то линеаризованная неоднородная система (8) имеет ограниченное (частное) решение $\widetilde{\mathbf{Y}}(\theta)$ тогда и только тогда, когда выполняются три условия ортогональности

$$
\int_{0}^{\Theta} W_{i}[\mathbf{H}] \stackrel{0}{R_{1}} \stackrel{0}{R_{2}} \stackrel{0}{R_{3}}(\theta) d \theta=0, \quad i=3,4,5 .
$$

Общее ограниченное решение содерэит три произвольные константы

$$
\mathbf{Y}(\theta)=\tilde{\mathbf{Y}}(\theta)+\sum_{i=0}^{2} \mathscr{C}_{i} \mathbf{Y}_{i}(\theta) \quad \forall \mathscr{C}_{i}=\text { const }
$$


Доказательство сводится к идентификации растущих (секулярных) слагаемых в интеграле (11). Условия (12) обеспечивают исключение таких членов.

Секулярные слагаемые в решении могут возникать лишш при совпадении частот правой части $\mathbf{H}(\theta)$ с собственными частотами $m \Omega, m \in \mathbb{Z}$, базисных решений. В том случае, когда такого совпадения нет, решение будет ограничено при дополнительном условии отсутствия малых знаменателей в соответствующих рядах Фурье. В частности, справедливо

СлЕДСТВИЕ 2. Пусть вектор-функиия правой части $\mathbf{H}(\theta)$ является гладкой, условно периодической функиией и ее разложение Фурье

$$
\mathbf{H}(\theta)=\sum_{m, p, q, r} \mathbf{H}_{m, p, q, r} \exp \left(i \theta\left[m \Omega+p\left(\Omega_{1}+\omega_{1}\right)+q\left(\Omega_{2}+\omega_{2}\right)+r\left(\Omega_{3}+\omega_{3}\right)\right]\right)
$$

содержит конечное число гармоник на дополнительных частотах: $|p|,|q|,|r| \leqslant$ $M<\infty$. Если выполнены условия (12), то общее условно периодическое решение имеет разлохсение Фурье такого жсе типа и содержит три произвольные константы $\mathscr{C}_{j}$ в форме (13).

3. Асимптотика ограниченных на бесконечности решений. Исходные неавтономные уравнения (1), называемые далее возмущенными, в общем случае не интегрируются. Для их исследования используется предъявленное выше невозмущенное решение (4). Оно берется в качестве главного (неубьвающего) члена асимптотики возмущенного решения на бесконечности. В получаемой таким образом конструкции правая часть уравнений (1) проявляется лишь в поправках порядка $\mathscr{O}\left(\theta^{-1}\right)$ при том, что амплитуды возмущений $f_{j}(\theta)$ вовсе не убьвают на бесконечности ${ }^{1}$. Эффект слабого влияния правых частей уравнений объясняется быстрыми осцилляциями рассматриваемого возмущения (1) с заданными фазовьгми функциями $\varphi_{j} \approx \theta^{2}, \theta \rightarrow \infty$.

ТЕоремА 4. Пусть значения параметров $\mathbf{E}$ не принадлежат плоскостям $E_{1}=0, E_{2}=E_{3} \quad$ u $\mathbf{E} \notin \mathscr{E}_{2} \cup \mathscr{E}_{0}$. Для уравнений (1) существует шестипараметрическое решение $\rho_{j}, \psi_{j}(\theta ; \mathbf{E}, \mathbf{S}), j=1,2,3$, которое для любого $N \geqslant 1$ имеет асимптотическое разлохсение на бесконечности

$$
\begin{aligned}
& \rho_{j}=\stackrel{0}{R}_{j}\left(\theta+S_{0} ; \mathbf{E}\right)+\sum_{n=1}^{N-1} \theta^{-n} \stackrel{n}{R}_{j}\left(\theta^{2}, \theta ; \mathbf{E}, \mathbf{S}\right)+\mathscr{O}\left(\theta^{-N}\right) \\
& \psi_{j}=\stackrel{0}{\Psi}_{j}\left(\theta+S_{0} ; \mathbf{E}\right)+\theta \Omega_{j}(\mathbf{E})+S_{j}+\sum_{n=1}^{N-1} \theta^{-n} \stackrel{n}{\Psi}_{j}\left(\theta^{2}, \theta ; \mathbf{E}, \mathbf{S}\right)+\mathscr{O}\left(\theta^{-N}\right)
\end{aligned}
$$

при $S_{1}+S_{2}+S_{3}=0$. Коэффичиенты $\stackrel{n}{\Psi}, \stackrel{n}{R}(\eta, \theta ; \mathbf{E}, \mathbf{S})$ являются условно периодическими функииями по переменным $\eta, \theta$. Вне резонансных поверхностей $\mathbf{E} \notin \mathscr{E}_{N+2} \cup \mathscr{E}_{0}$ оценки остатков асимптотики являются равномерными по всем параметрам на любом компакте.

\footnotetext{
${ }^{1}$ Надо иметь ввиду, что те же самые возмущенные уравнения (1) имеют растущие решения, асимптотика которых в главном определяется параметрами правых частей.
} 
СЛЕДСТВИЕ 3. Первые поправки выражаются через тригонометрические функиии и невозмущенное решение по формулам

$$
\begin{aligned}
& \stackrel{1}{R}_{j}=-\frac{f_{j}}{2 \alpha_{j}} \cos \left(\stackrel{0}{\Psi}_{j}+\left(\Omega_{j}+\omega_{j}\right) \theta+S_{j}+\alpha_{j} \theta^{2}\right) \\
& \stackrel{1}{\Psi}_{j}=\frac{f_{j}}{2 \alpha_{j}}\left(\stackrel{0}{R}_{j}\right)^{-1} \sin \left(\stackrel{0}{\Psi}_{j}+\left(\Omega_{j}+\omega_{j}\right) \theta+S_{j}+\alpha_{j} \theta^{2}\right) .
\end{aligned}
$$

Стариие поправки имеют подобную структуру в виде тригонометрических полиномов по “быстрым" переменным $\alpha_{j} \theta^{2}$.

Приводимое ниже доказательство теоремы представляет собой описание процедуры построения коэффициентов асимптотических рядов. Обоснование асимптотики с оценкой остаточных членов может быть вьполнено аналогично [17] и здесь не приводится. Построение коэффициентов сводится к решению дифференциальных уравнений и состоит из нескольких этапов. На разньх этапах находится зависимость от разных фазовых переменных, что можно интерпретировать, как разделение движений с разными временными масштабами. В асимптотическом решении идентифищируются три масштаба, характерные для далеких времен $\theta \rightarrow \infty$. Самьй быстрый масштаб задается быстрьми вьнуждающими колебаниями с фазами $\varphi_{j}(\theta) \approx \theta^{2}, \theta \rightarrow \infty$. Второй масштаб определяется собственными колебаниями невозмущенной системы с частотами $\Omega, \Omega_{j}$. Третий (медленный) масштаб характеризуется обратной степенью $\theta^{-1}$. Процедуру получения дифференциальных уравнений для коэффищиентов асимптотики на каждом последующем (медленном) масштабе можно рассматривать, как усреднение на предыдущем (быстром) масштабе.

3.1. Усреднение в масштабе внешней накачки. С формальной точки зрения первый этап построения асимптотики состоит в замене переменных, которая вьполняется в асимптотическом приближении.

Вводится дополнительная переменная $\eta=\theta^{2} / 2$. Решение ищется в виде функций нескольких переменных: $\rho_{j}=\widetilde{\rho}_{j}(\eta, \theta ; \mathbf{Y}), \psi_{j}=\widetilde{\psi}_{j}(\eta, \theta ; \mathbf{Y})$. Фигурирующий здесь вектор $\mathbf{Y} \in \mathbb{R}^{6}$ рассматривается, как новьй набор неизвестных функций $\mathbf{Y}=\left\{R_{j}(\theta), \Psi_{j}(\theta)\right\}^{\top}$, а введенные соотношения интерпретируются, как замена переменных. Требуется подыскать функции замены $\widetilde{\rho}_{j}, \widetilde{\psi}_{j}$ так, чтобы уравнения для $\mathbf{Y}$ не содержали зависимость от $\eta=\theta^{2} / 2$ в осциллируюших частях.

Поставленная таким образом задача решается не в точной постановке, а в асимптотическом приближении при $\theta \rightarrow \infty$. С этой целью вводится асимптотический анзатц, аналог анзатца Крылова-Боголюбова:

$$
\left\{\rho_{j}(\theta), \psi_{j}(\theta)\right\}^{\top}=\sum_{n=0}^{\infty} \theta^{-n} \mathbf{y}(\eta ; \mathbf{Y}), \quad \theta \rightarrow \infty
$$

Требуется определить коэффициенты $\stackrel{n}{\mathbf{y}}(\eta ; \mathbf{Y})$ в классе вектор-функций, ограниченных по $\eta \in \mathbb{R}$ так, чтобы исходные уравнения (1) перешли в систему

$$
\frac{d \mathbf{Y}}{d \theta}=\mathbf{F}(\mathbf{Y}, \theta)
$$

в которой правая часть представляется в виде асимптотического ряда

$$
\mathbf{F}=\sum_{n=0}^{\infty} \theta^{-n} \mathbf{F}(\mathbf{Y}), \quad \theta \rightarrow \infty
$$


Лемма 1. Из требования асимптотического выполнения уравнений (1) коэффичиенты рядов (16), (18) определяются однозначно по рекуррентным формулам. Они представляют собой условно периодические функиии по $\eta$ с тремя независимымми частотами $\alpha_{j}$. Получаемая при этом система (17) оказывается асимптотически әквивалентна исходным уравнениям (1).

ДокАЗАТЕЛЬСТво. После подстановки асимптотического ряда (16) в уравнения (1) и приравнивания выражений при одинаковых степенях $\theta$ получаются уравнения для коэффициентов $\stackrel{n}{\mathbf{y}}(\eta ; \mathbf{Y})$, которые вьглядят тривиально

$$
\partial_{\eta} \stackrel{n}{\mathbf{y}}=\stackrel{n}{\mathbf{f}}(\eta ; \mathbf{Y})
$$

Правые части на каждом шаге определяются через предыдущие приближения. На исходном шаге уравнения оказываются однородными $\mathbf{f} \equiv 0$. Поэтому главньй член асимптотического решения не зависит от $\eta$, и без ограничения обшности можно положить $\stackrel{0}{\mathbf{y}} \equiv \mathbf{Y}$.

Во всех последующих приближениях зависимость от $\eta$ входит в виде комбинаций тригонометрических функций. Дополнительные условия ограниченности по $\eta$ сводятся к требованию обращения в нуль средних значений от правых частей $\langle\stackrel{n}{\mathbf{f}}\rangle=0$. Как раз эти требования приводят к определению коэффициентов $\stackrel{n}{\mathbf{F}}(\mathbf{Y})$. В частности, при решении задачи для первой поправки $\stackrel{1}{\mathbf{y}}$ находится $\stackrel{0}{\mathbf{F}}=-\mathbf{L}(\mathbf{Y})$.

Отметим, что при интегрировании уравнений в быстрой переменной $\eta$ не возникает проблемы малых знаменателей, характерной для многомерньх систем, поскольку получаемые здесь разложения Фурье представляют собой тригонометрические полиномы, а не бесконечные ряды.

В заключение вьпишем формулы, которые дают выражения для правых частей усредненных уравнений. Первая поправка оказьвается нулевой $\stackrel{1}{\mathbf{F}} \equiv 0$. Вторая поправка представима в виде суммы двух слагаемых, из которых первое определяется через нелинейную часть невозмущенной системы

$$
\stackrel{2}{\mathbf{F}}(\mathbf{Y})=\lambda \mathbf{L}(\mathbf{Y})+\mathbf{G}(\mathbf{Y}), \quad \lambda=\sum_{j=1}^{3}\left(\frac{f_{j}}{8 \alpha_{j} R_{j}}\right)
$$

Вектор второго слагаемого содержит компоненты, из которых ненулевыми могут быть лишь последние три: $\mathbf{G}=\left\{0,0,0, F_{\psi, 1}, F_{\psi, 2}, F_{\psi, 3}\right\}^{\top}$ при

$F_{\psi, j}=\gamma_{j} \frac{2}{R_{j}^{2}}\left(R_{l}\left\langle\rho_{j} \rho_{k}\right\rangle+R_{k}\left\langle\rho_{j} \rho_{l}\right\rangle-\frac{R_{k} R_{l}}{R_{j}} \frac{f_{j}^{2}}{8 \alpha_{j}^{2}}\right) \cos \Psi, \quad j+k+l=6, j=1,2,3, k \neq l$.

Эти формулы содержат средние (по $\eta$ ) значения от произведений первьх поправок

$$
\stackrel{1}{\rho}_{j}(\eta ; \mathbf{Y})=-\frac{f_{j}}{2 \alpha_{j}} \cos \left(\Psi_{j}+\omega_{j} \theta+\alpha_{j} \eta\right)
$$

Поэтому среди первьх двух слагаемых встречаются ненулевые лишь в резонансном случае, когда совпадает хотя бы пара "быстрых" частот в вынуждающих колебаниях: $\exists p \neq q: \alpha_{p}^{2}=\alpha_{q}^{2}$. Таким способом получаются векторы $\stackrel{n}{\mathbf{F}}(\mathbf{Y})$ на любом шаге. Лемма доказана. 
3.2. Усреднение в масштабе свободных колебаний. Асимптотическое интегрирование усредненных уравнений (17) составляет второй этап построения асимптотики решения исходных уравнений (1). По своей форме усредненная система мало отличается от исходной. В самом деле, если главньй член разложения $\mathbf{F}(\mathbf{Y} ; \theta)$ перенести в левую часть, то получим уравнения, которые совпадают по форме с (1)

$$
\begin{gathered}
\frac{d R_{j}}{d \theta}+\gamma_{j} R_{k} R_{l} \sin \Psi=F_{\rho, j}(\mathbf{Y}, \theta), \quad \frac{d \Psi_{j}}{d \theta}+\gamma_{j}\left(R_{k} R_{l} / R_{j}\right) \cos \Psi=F_{\psi, j}(\mathbf{Y}, \theta) \\
\Psi=\Psi_{1}+\Psi_{2}+\Psi_{3}, \quad j=1,2,3, \quad j+k+l=6, \quad k \neq l
\end{gathered}
$$

Основное отличие содержится в векторе правых частей $\widetilde{\mathbf{F}}=\left\{F_{\rho, j}, F_{\psi, j}\right\}^{\top}$, которьй в силу (18) имеет разложение по отрицательным степеням:

$$
\widetilde{\mathbf{F}}=\sum_{n=2}^{\infty} \theta^{-n} \mathbf{F}(\mathbf{Y}), \quad \theta \rightarrow \infty
$$

и не содержит каких-либо осцилляций.

Поскольку правая часть стремится к нулю при $\theta \rightarrow \infty$, ясно, что главную роль в асимптотике играет решение невозмущенной системы уравнений $\stackrel{0}{\mathbf{Y}}(\theta ; \mathbf{E}, \mathbf{S})$. На его основе можно построить асимптотическое решение в виде ряда по отрицательным степеням $\theta^{-n}$ с коэффициентами, которые вычисляются в терминах вектор-функции $\stackrel{0}{\mathbf{Y}}(\theta$; $\mathbf{E}, \mathbf{S})$. Однако прямое конструирование асимптотики в такой, наиболее простой, форме наталкивается на трудности при исключении секулярных слагаемых. Трудности связаны с исследованием возникающих систем нелинейных алгебраических уравнений. Эти проблемы можно обойти, если использовать идеи Крылова-Боголюбова-Кузмака. Для асимптотического решения вводится анзатц

$$
\mathbf{Y}(\theta)=\stackrel{0}{\mathbf{Y}}(\theta ; \mathbf{E}, \mathbf{S})+\sum_{n=1}^{\infty} \theta^{-n} \stackrel{n}{\mathbf{Y}}(\theta ; \mathbf{E}, \mathbf{S})
$$

коэффищиенты которого содержат дополнительную зависимость от $\theta$ в сдвигах фаз: $S_{j}=$ $S_{j}(\theta), j=0,1,2, S_{1}+S_{2}+S_{3} \equiv 0$; параметры $\mathbf{E}$ остаются постоянными. В этом асимптотическом решении требуется определить коэффициенты $\stackrel{n}{\mathbf{Y}}(\theta ; \mathbf{E}, \mathbf{S})$ в классе функций, ограниченных по быстрой переменной $\theta \in \mathbb{R}$. Требования ограниченности будут удовлетворяться за счет подходящего выбора вектора $\mathbf{S}(\theta)=\left\{S_{0}(\theta), S_{1}(\theta), S_{2}(\theta)\right\}$. Для этих функций фазовых сдвигов вводятся уравнения, которые являются отдаленными аналогами уравнений эйконала:

$$
\frac{d \mathbf{S}}{d \theta}=\mathbf{Z}(\theta, \mathbf{S} ; \mathbf{E})
$$

На первом этапе будут получены не сами сдвиги $\mathbf{S}$, а правые части уравнений в виде асимптотических рядов

$$
\mathbf{Z}(\theta, \mathbf{S} ; \mathbf{E})=\sum_{n=1}^{\infty} \theta^{-n} \stackrel{n}{\mathbf{Z}}(\mathbf{S}, \mathbf{E}) .
$$

Асимптотика для вектора $\mathbf{S}(\theta)$ будет строиться на втором этапе. 
Лемма 2. Пусть значения параметров $\mathbf{E}$ не принадлежат плоскостям $E_{1}=0$, $E_{2}=E_{3}$. Тогда из требования асимптотического выполнения уравнений (20) коәффициенты рядов (22), (24) определяются по рекуррентным формулам. Векторы $\stackrel{n}{\mathbf{Y}}(\theta ; \mathbf{E}, \mathbf{S})$ представляют собой условно периодические функиии по ө с периодами $\Omega, \Omega_{j}+\omega_{j}$. Структура рядов (24) в стариих поправках меняется при выходе параметров $\mathbf{E}$ на резонансные поверхности.

ДокАЗАтЕльство. Поправки $\stackrel{n}{\mathbf{Y}}(\theta ; \mathbf{E}, \mathbf{S})$ определяются из линеаризованных уравнений (8), которые для краткости запишем в векторной форме

$$
\frac{d{ }^{\mathbf{Y}}}{d \theta}+L_{1}(\stackrel{0}{\mathbf{Y}}) \stackrel{n}{\mathbf{Y}}=\stackrel{n}{\mathbf{H}}(\theta ; \mathbf{S}, \mathbf{E})
$$

Переменные $\mathbf{S}, \mathbf{E}$ присутствуют здесь в качестве параметров; в частности, от них зависит линеаризованная часть оператора $L_{1}(\stackrel{0}{\mathbf{Y}}(\theta ; \mathbf{S}, \mathbf{E}))$. Правые части $\stackrel{n}{\mathbf{H}}$ на разных шагах $n=1,2,3, \ldots$ вычисляются по рекуррентным формулам через предыдушие приближения и содержат три неопределенные компоненты вектора $\mathrm{n}_{\mathbf{Z}}=\left\{\stackrel{n}{Z}_{0}, \stackrel{n}{Z}_{1}, \stackrel{n}{Z}_{2}\right\}$ в комбинации с базисными решениями $\mathbf{Y}_{i}$. В частности,

$$
\begin{gathered}
\stackrel{1}{\mathbf{H}}=-\sum_{i=0}^{2} \stackrel{1}{Z}_{i} \mathbf{Y}_{i}, \quad \stackrel{2}{\mathbf{H}}=\stackrel{1}{\mathbf{Y}}-L_{2}(\stackrel{0}{\mathbf{Y}}) \stackrel{1}{\mathbf{Y}} \stackrel{1}{\mathbf{Y}}-\sum_{i=0}^{2}\left[\stackrel{2}{Z}_{i} \mathbf{Y}_{i}+\stackrel{1}{Z}_{i} \partial_{S_{i}} \stackrel{1}{\mathbf{Y}}\right]+\stackrel{2}{\mathbf{F}}, \\
\stackrel{n+1}{\mathbf{H}}=n \stackrel{n}{\mathbf{Y}}-2 L_{2}(\stackrel{0}{\mathbf{Y}}) \stackrel{1}{\mathbf{Y}} \stackrel{n}{\mathbf{Y}}-\sum_{i=0}^{2}\left[\stackrel{n+1}{Z}_{i} \mathbf{Y}_{i}+\stackrel{1}{Z}_{i} \partial_{S_{i}} \stackrel{n}{\mathbf{Y}}\right]+\stackrel{n+1}{\mathbf{F}}+\stackrel{n}{\mathbf{G}}(\stackrel{0}{\mathbf{Y}}, \ldots, \stackrel{n-1}{\mathbf{Y}}), \quad n \geqslant 2 .
\end{gathered}
$$

Через $L_{2}$ здесь обозначен оператор квадратичной формы, который получается при разложении Тейлора исходного нелинейного оператора. В векторе $\stackrel{n}{\mathbf{G}}$ перечислена зависимость от предыдущих приближений.

Решения линеаризованных уравнений вьписьваются через интегралы в терминах вектора $\stackrel{0}{\mathbf{Y}}(\theta ; \mathbf{S}, \mathbf{E})$. Требования ограниченности (12) удовлетворяются за счет произвола в $\stackrel{n}{Z}_{i}$. При этом выбор $\stackrel{n}{Z}_{i}$ затягивается на один шаг. Так, на первом шаге $n=1$ условия (12) выполняются автоматически в силу специфики правой части $\stackrel{1}{\mathbf{H}}$. Ограниченное решение выписывается через осциллируюшие слагаемые растущих базисных решений

$$
\stackrel{1}{\mathbf{Y}}=\sum_{i=0}^{2} \stackrel{1}{Z}_{i} \sum_{m=3}^{5} \mu_{i, m} \widehat{\mathbf{Y}}_{m}
$$

здесь $\left\{\mu_{i, m}\right\}$ - матрица, обратная к $\left\{\nu_{i, m}\right\}$.

Уравнения для $\stackrel{1}{Z}_{i}$ возникают на втором шаге из требований $(12)$ и оказьваются линейньми:

$$
\sum_{i=0}^{2} \mu_{i, m} \stackrel{1}{Z}_{i}=\left\langle W_{m}[\stackrel{2}{\mathbf{F}}] / W\right\rangle, \quad m=3,4,5 .
$$


Самое замечательное, что в этих соотношениях не проявляются квадратичныепо $\stackrel{1}{\mathbf{Z}}$ сла-

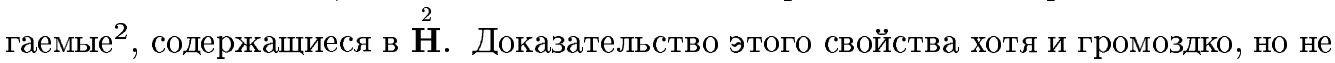
содержит принципиальных трудностей.

Выражения в правой части (27) представляют собой средние значения по $\theta$ известных функций. Решение алгебраических уравнений $\stackrel{1}{Z}_{i}=\stackrel{1}{Z}_{i}(\mathbf{S}, \mathbf{E})$ выписьвается в явной форме. После этого можно выписать ограниченное решение линеаризованной системы $\stackrel{2}{\mathbf{Y}}(\theta ; \mathbf{S}, \mathbf{E})$. Оно содержит неопределенные коэффициенты ${ }_{Z}^{2}$ в форме, аналогичной (26). Эти коэффищиенты находятся на следующем шаге из алгебраических уравнений типа (27). Важно отметить, что средние значения в правых частях алгебраических уравнений крайне чувствительны к вариации параметров $\mathbf{E}$ и меняются скачком при выходе $\mathbf{E}$ на резонансные поверхности. Множество резонансных поверхностей расширяется с ростом номера приближения. При любом фиксированном $\mathbf{E}$ полное построение рядов (22), (24) вьполняется по индукции. Лемма доказана.

После того, как построены ряды правых частей (24), можно переходить к решению уравнений для фаз (23) в асимптотическом приближении при $\theta \rightarrow \infty$. Коэффициенты рядов (24) являются гладкими функциями по $\mathbf{S}$, поэтому асимптотическое интегрирование уравнений не вызьвает затруднений. Однако зависимость их по Е оказьвается крайне нерегулярной из-за резонансов. Например, первьй коэффициент $\mathbf{Z}(\mathbf{S}, \mathbf{E})$ может содержать компоненты, отличные от нуля лишш на резонансной поверхности главного порядка $\mathscr{E}_{2}$. Для таких значений параметров фазовые сдвиги $\mathbf{S}(\theta)$ в главных членах асимптотики могут содержать логарифмы $\ln \theta$, как это случается, например, в одномерных системах, либо дробные степени, что определяется структурой $\stackrel{1}{\mathbf{Z}}(\mathbf{S}, \mathbf{E})$. Если же из множества параметров $\mathbf{E}$ исключить резонансные поверхности главного порядка, то фазовые сдвиги $\mathbf{S}(\theta)$ разлагаются по целым отрицательным степеням.

Лемма 3. Пусть значения параметров $\mathbf{E}$ не принадлежсат плоскостям $E_{1}=0$, $E_{2}=E_{3}$ и $\mathbf{E} \notin \mathscr{E}_{2} \cup \mathscr{E}_{0}$. Тогда существует асимптотическое решение уравнений (23), представимое в виде ряда

$$
\mathbf{S}(\theta ; \mathbf{E})=\stackrel{0}{\mathbf{S}}+\sum_{n=1}^{\infty} \theta^{-n} \stackrel{n}{\mathbf{S}}(\mathbf{E}), \quad \theta \rightarrow \infty
$$

с тремя произвольными параметрами $\stackrel{0}{\mathbf{S}}=\left\{\stackrel{0}{S}_{0}, \stackrel{0}{S}_{1}, \stackrel{0}{S}_{2}\right\}$. Структура рядов в старших поправках меняется при выходе параметров $\mathbf{E}$ на резонансные поверхности.

ДокАЗАТЕЛЬСтво. Вне резонансной поверхности $\mathscr{E}_{2}$ уравнения (27) оказьваются однородньми и, следовательно, $\stackrel{1}{\mathbf{Z}}=0$. После этого построение асимптотического решения уравнений (23) в виде ряда (28) сводится к последовательному определению коэффициентов $\stackrel{n}{\mathbf{S}}(\mathbf{E})$ из рекуррентных формул. Вектор $\stackrel{0}{\mathbf{S}}=$ const играет роль константы интегрирования.

\footnotetext{
${ }^{2}$ В этом месте обнаруживается преимущество выбранного анзатца с переменными сдвигами фаз.
} 
СлЕДСТВИЕ 4. Ряды (22), (23) представляют асимптотическое решение для усредненных уравнений (20) при указанных значениях $\mathbf{E}$.

Объединение последнего утверждения с леммой 1 заканчивает формальную часть доказательства теоремы 4. Требуемое разложение типа (14) в виде бесконечного ряда получается из рядов $(16),(22),(23)$ путем переразложения коэффициентов. Первая поправка в форме $(15)$ выделяется с учетом тождества $\stackrel{1}{\mathbf{Y}} \equiv 0$, которое получается в силу $\stackrel{1}{\mathbf{Z}}=0$. Структура рядов зависит от параметров $\mathbf{E}$.

\section{СПИСОК ЦИТИРОВАННОЙ ЛИТЕРАТУРЫ}

[1] Бломберген Н. Нелинейная оптика. М.: Мир, 1966.

[2] Каплан Ф.Е., Кривцов Ю. А., Рылов В. А. Параметрические генераторы и делители частоты. М.: Советское радио, 1966.

[3] Наугольных К. А., Островский Л. А. Нелинейные волновые процессы в акустике. М.: Наука, 1990.

[4] Боголюбов Н. Н., Митропольский Ю. А. Асимптотические методы в теории нелинейных колебаний. М.: Наука, 1974.

[5] Yariv Sh., Friedland L. Autoresonant interaction of three nonlinear adiabatic oscillators // Phys. Rev. E. 1993. V. 48. № 4. P. 3072-3076.

[6] Rokni U., Friedland L. Double autoresonance in two-dimensional dynamical systems // Phys. Rev. E. 1939. V. 59. № 5. P. 5242-5252.

[7] Заславский Г. М., Сагдеев Р.З. Введение в нелинейную физику. От маятника до турбулентности и хаоса. М.: Наука, 1977.

[8] Fajans J., Friedland L. Autoresonant (nonstationary) excitation of a pendulum, Plutinos, plasmas and other nonlinear oscillators // Amer. J. Phys. 2001. V. 69. №10. P. 1096-1102.

[9] Калякин Л.А. Асимптотический анализ модели авторезонанса // Докл. РАН. 2001. T. 378. № 5. C. 594-597.

[10] Калякин Л.А.Асимптотика решений уравнений главного резонанса на бесконечности // Докл. РАН. 2003. Т. 388. № 3. С. 305-308.

[11] Козлов В.В., Фурта С. Д. Асимптотики решений сильно нелинейньх систем дифференциальных уравнений. М.: Изд-во Московского ун-та, 1996.

[12] Брюно А. Д. Степенная геометрия в алгебраических и дифференциальных уравнениях. М.: Наука, 1998.

[13] Кузмак Г. Е. Асимптотические решения нелинейных дифференциальных уравнений с переменными коэффициентами // ПММ. 1951. Т. 23. № 3. С. 519-506.

[14] Федорюк М. В. Метод ВКБ для нелинейного уравнения второго порядка // ЖВМиМФ. 1986. Т. 26. № 2. C. 196-210.

[15] Арнольд В. И. Малые знаменатели и проблемы устойчивости движения в классической и небесной механике // УМН. 1963. Т. 18. №6. С. 91-192.

[16] Арнольд В.И. Дополнительные главы теории обыкновенных дифференциальных уравнений. М.: Наука, 1978.

[17] Kalyakin L. A. Justification of asymptotic expansion for the principal resonance equations // Proc. Steklov Inst. Math. Suppl. 1. 2003. P. S108-S122.

Институт математики с ВЦ РАН, г. Уфа

Поступило

E-mail: klenru@mail.ru, yulya@mail.rb.ru

20.05 .2004

Исправленный вариант

17.12 .2004 